\title{
Relation of QT-Interval Variability to Ventricular Arrhythmias During Percutaneous Transluminal Coronary Angioplasty
}

\author{
Akio Kajiyama, MD; Daiji Saito, MD; Takashi Murakami, MD**; \\ Teruo Shiraki, MD; Takefumi Oka, MD; Masayuki Doi, MD; \\ Takuro Masaka, MD; Kazuo Tanemoto, MD*; Takao Tsuji, MD**
}

\begin{abstract}
The present study investigated the role of the dispersion of QT interval in percutaneous transluminal coronary angioplasty (PTCA)-induced ventricular tachyarrhythmias. Patients with effort angina without a previous myocardial infarction $(n=22)$, who had single-vessel disease of the anterior descending coronary artery (LAD), underwent PTCA if the coronary lesion was 75\% or more stenosed in segment 6 or 7 of the LAD. The standard 12-lead ECG was continuously recorded during the procedure. Averaged QTc and QTac intervals, where QTac was the interval from the beginning of QRS complex to the nadir of T wave corrected by Bazett's formula, did not change significantly during PTCA. Of the 22 patients, 7 showed ventricular arrhythmias during PTCA. The maximum difference $(\Delta \mathrm{QTc})$ and the standard deviation $(\mathrm{QTcSD})$ of the corrected QT interval in the standard 12-lead ECG increased significantly during PTCA in the 7 patients with ventricular arrhythmias, whereas they decrreased in the 15 patients without ventricular arrhythmias. $\triangle$ QTac and QTacSD were not affected by PTCA regardless of ventricular arrhythmias, which shows that the increases in the variation of the ventricular repolarization process play a role in PTCA-induced ventricular arrhythmias. (Jpn Circ J 2001; 65: 779-782)
\end{abstract}

Key Words: Percutaneous transluminal coronary angioplasty (PTCA); QT dispersion; Stable angina pectoris; Ventricular tachyarrhythmias

$\mathbf{T}$ here is variation of repolarization across the ventricular myocardium in normal ${ }^{1}$ and diseased hearts,-4 and there is strong experimental evidence linking the vulnerability of ventricular myocardium to arrhythmias with increased dispersion of refractoriness. This variation in the recovery time is widely accepted as an important factor in the pathogenesis of ventricular arrhythmias because it increases the vulnerability to ventricular re-entry. Previous work has shown that the QT interval varies from lead to lead of the surface ECG, creating QT dispersion, which represents the regional difference in repolarization!,2,5

Ventricular arrhythmias are frequently observed during balloon inflation for percutaneous transluminal coronary angioplasty (PTCA) and this study investigated the relation of the QT interval dispersion to PTCA-induced ventricular arrhythmias.

\section{Methods}

\section{Patient Selection}

Inclusion criteria for this study were (1) angina pectoris (typical chest pain within 2 weeks of enrolment; transient ischemic ST depression $\geq 0.1 \mathrm{mV}$ during chest pain), (2) stable on conventional medication, (3) angiographically significant stenosis $(\geq 75 \%)$ in segment 6 or 7 of the anterior

(Received August 4, 2000; revised manuscript received June 11, 2001; accepted June 13, 2001)

Departments of Cardiology and *Cardiovascular Surgery, Iwakuni National Hospital, Iwakuni and **The First Department of Internal Medicine, Okayama University Medical School, Okayama, Japan

Mailing address: Akio Kajiyama, MD, The Department of Cardiology, Iwakuni National Hospital, 2-5-1 Kuroiso, Iwakuni, Yamaguchi 7408510, Japan descending coronary artery, and (4) candidate for elective PTCA. From September 1994 through December 1995, 51 consecutive patients with stable effort angina were admitted to hospital for PTCA and of these, 26 patients met the study criteria. The remaining 25 were diagnosed as postmyocardial infarction angina, and were excluded because of their previously damaged myocardium. The remaining 26 patients had normal left ventricular function on cardiac ultrasound examination and/or left ventriculogram. Four other patients were excluded: 3 with atrial fibrillation and 1 with complete right bundle branch block.

\section{Study Protocol}

Two days prior to angiography, all patients were weaned from their medications except for sublingual nitroglycerin. Coronary angioplasty was performed using the Judkins technique. Hydroxyzine pamoate $(25 \mathrm{mg})$ was administered subcutaneously $30 \mathrm{~min}$ before the procedure as a tranquillizer. Sodium heparin $(10,000$ unit) was injected intrave-

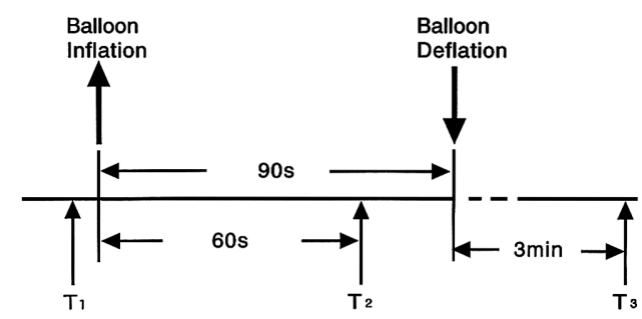

Fig 1. Study protocol for PTCA-induced ventricular arrhythmias. The standard 12-lead ECG was continuously recorded throughout the procedure at a paper speed of $25 \mathrm{~mm} / \mathrm{s}$. At T1, T2, and T3 the paper speed was increased to $50 \mathrm{~mm} / \mathrm{s}$. 
Table 1 Background Data and QTc Before PTCA

\begin{tabular}{lcc}
\hline \hline & Arrhythmia $(+)$ & Arrhythmia $(-)$ \\
\hline No. of patients & 7 & 15 \\
Age (years) & $72 \pm 7$ & $63 \pm 4$ \\
M:F & $5: 2$ & $13: 2$ \\
BPs (mmHg) & $134 \pm 9$ & $136 \pm 11$ \\
Involved seg (\#6:\#7) & $4: 3$ & $7: 8$ \\
RR interval (s) & $0.88 \pm 0.16$ & $0.87 \pm 0.14$ \\
OTc interval (s) & & \\
$I$ & $0.43 \pm 0.05$ & $0.39 \pm 0.03$ \\
II & $0.45 \pm 0.03$ & $0.43 \pm 0.04$ \\
III & $0.43 \pm 0.02$ & $0.43 \pm 0.04$ \\
$a V_{R}$ & $0.44 \pm 0.02$ & $0.43 \pm 0.04$ \\
$a V_{L}$ & $0.43 \pm 0.03$ & $0.43 \pm 0.03$ \\
$a V_{F}$ & $0.44 \pm 0.03$ & $0.43 \pm 0.04$ \\
$V_{1}$ & $0.44 \pm 0.04$ & $0.42 \pm 0.03$ \\
$V_{2}$ & $0.44 \pm 0.03$ & $0.43 \pm 0.03$ \\
$V_{3}$ & $0.44 \pm 0.03$ & $0.44 \pm 0.03$ \\
$V_{4}$ & $0.45 \pm 0.04$ & $0.44 \pm 0.04$ \\
$V_{5}$ & $0.45 \pm 0.04$ & $0.44 \pm 0.04$ \\
$V_{6}$ & $0.44 \pm 0.04$ & $0.43 \pm 0.04$ \\
\end{tabular}

BPs, systolic blood pressure; Involved seg (\#6:\#7), involved segment of the anterior descending coronary artery. No significant difference of age, $R R$ interval and QTC of any leads between the patients with and without ventricular arrhythmias.

nously just before the catheterization. Sublingual nitroglycerin was given before the guiding catheter was positioned in the coronary ostium and every 30 min thereafter.

The balloon was inflated in the anterior descending coronary artery for $90 \mathrm{~s}$, unless the patient experienced severe chest pain, or hemodynamic instability $(0 / 22)$. The standard 12-lead ECG was continuously recorded throughout the procedure. At T1 (before balloon inflation), T2 (60 s after balloon inflation) and T3 (3 min after balloon deflation), the paper speed was increased to $50 \mathrm{~mm} / \mathrm{s}$ for measurements and data were obtained from the first inflation (Fig 1). None of the patients within the study required antiarrhythmic medication during the PTCA procedure.

To investigate the relationship between the QT intervals and ventricular arrhythmias during PTCA, we divided the patients into 2 groups with respect to ventricular arrhythmias, and compared the repolarization parameters (QTc, $\Delta \mathrm{QTc}, \mathrm{QTac}$ and $\Delta \mathrm{QTac})$ of the 2 groups.

The study protocol was in agreement with the guidelines of the ethical committee of the institution and written informed consent was obtained from each patient.

\section{Data Analysis}

In each patient, 4 parameters were measured in 3 consecutive heart beats (QT, QTa and Bazett's heart rate corrected QTc and QTac) and averaged in each of the 12 ECG leads. QT and QTa were defined as the intervals from the beginning of QRS complex to the end of the T wave, and the top or nadir of the $\mathrm{T}$ wave, respectively, while excluding the $\mathrm{U}$ wave. Two parameters of repolarization dispersion were computed: (1) the maximum QTc dispersion $(\Delta \mathrm{QTc})$, which was defined as the difference between the maximum and minimum QT interval measurements occurring in any of the 12 leads on a standard ECG, and the standard deviation among the measured QTc in all the leads. All measurements were taken from the ECG segment prior to any arrhythmias.

Analysis of variance and Bonferroni's modified t-test were used to comparing the data within a patient group, and Student's t-test compared the data between the patients who experienced ventricular arrhythmia and those which did not.
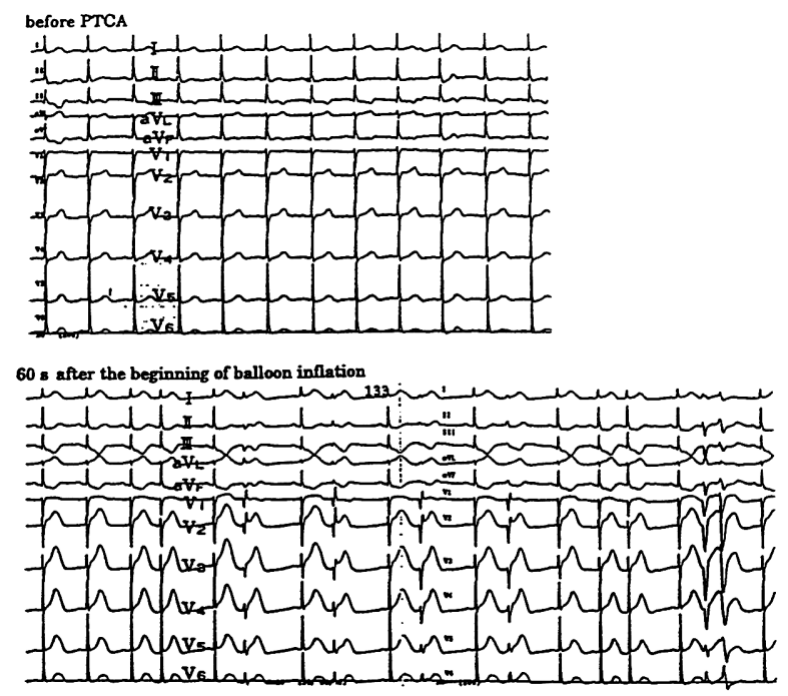

Fig 2. Representative ECG with a double PVC.

\section{Results}

Twenty-two patients with angina pectoris without previous myocardial infarction were enrolled; their background data, baseline heart rate and QT intervals are presented in Table 1. All patients had significant coronary stenosis in segment 6 or 7 of the anterior descending coronary artery, and both the ${ }^{201} \mathrm{Tl}$ myocardial scintigram and ECG showed exercise-induced transient myocardial ischemia in the anterior wall of the left ventricle. No patient showed abnormal Q waves or significant arrhythmias on the ECG before balloon inflation. The mean age of the patients was 66 years; 18 were men and 4 were women.

With balloon inflation, the ST segments in leads $\mathrm{V}_{2-5}$ elevated by $2.0 \mathrm{~mm}(0.2 \mathrm{mV})$ or more compared with the pre-inflation ST level. Seven patients showed additional ST segment elevation in lead $\mathrm{V}_{1}$, and 4 patients in lead V6. The heart rate tended to increase with balloon inflation, but not to a significant level. The shape and duration of the QRS complex did not change with balloon inflation in any lead regardless of the elevation of the ST segment.

The averaged QTc tended to be shortened by balloon inflation, but this was not statistically significant in any of the 12 leads. The maximum ST-segment elevation was seen in lead $V_{3}$ in over two-thirds $(16 / 22)$ of the patients. The QTc in lead V3 was slightly but insignificantly decreased during balloon inflation (Table 1 ). $\triangle$ QTc reduced from $0.058 \pm 0.016$ to $0.045 \pm 0.018$ with balloon inflation, but again, this was not significant $(\mathrm{p}<0.1)$. The average $\mathrm{ST}$ segment $3 \mathrm{~min}$ after balloon deflation, the QTc interval tended to return to the pre-inflation value with a slight decrease in heart rate. The post-inflation $\Delta \mathrm{QTc}$ did not significantly change from the $\triangle \mathrm{QT} c$ during PTCA. The standard deviation of QTc of the 12 leads insignificantly decreased during balloon inflation, and returned to the preinflation level $3 \mathrm{~min}$ after the deflation of the balloon. The balloon inflation tended to shorten the QTac, but not significantly. QTac also showed a tendency to decrease during the balloon inflation and return to the pre-inflation value.

Of the 22 patients, 15 had no ventricular arrhythmias during balloon inflation, and 7 showed ventricular arrhythmias in the latter half of the balloon inflation period; 1 patient had a triple premature ventricular contraction (PVC), 
Table 2 Effects of Intracoronary Balloon Inflation on QTe and QTac in Lead $\mathrm{V}_{3}$

\begin{tabular}{lccc}
\hline \hline & Arrhythmia $(+)$ & Arrhythmia $(-)$ & $p$ value \\
\hline No. of patients & 7 & 15 & \\
QTc interval (s) & & & \\
Before inflation & $0.44 \pm 0.03$ & $0.44 \pm 0.03$ & $N S$ \\
$\quad$ During inflation & $0.46 \pm 0.04$ & $0.43 \pm 0.03$ & $N S$ \\
$\quad$ After inflation & $0.45 \pm 0.03$ & $0.44 \pm 0.03$ & $N S$ \\
QTac interval (s) & & & \\
Before inflation & $0.34 \pm 0.05$ & $0.31 \pm 0.03$ & $N S$ \\
During inflation & $0.32 \pm 0.02$ & $0.32 \pm 0.02$ & $N S$ \\
After inflation & $0.34 \pm 0.03$ & $0.31 \pm 0.04$ & $N S$
\end{tabular}

Intracoronary balloon inflation did not cause a significant effect on averaged QTC and QTac regardless of the presence or absence of ventricular arrhythmias during PTCA.

3 had a double PVC followed by bigeminy lasting about 30 s, 2 had PVCs with bigeminy and 1 had single type PVCs. A representative record with a double PVC is shown in Fig 2. QTc tended to be longer in the patients with ventricular arrhythmias than the patients without before, during and after the termination of balloon inflation, but the difference did not reach a significant level in any leads in any phase of the study. At the end of balloon inflation, QTc in lead $V_{3}$ had a tendency to decrease in patients with ventricular arrhythmias, but the magnitude was not statistically significant (Table 2). QTc in patients without arrhythmias did not change during PTCA.

$\Delta$ QTc was not significantly different between the 2 groups before the balloon inflation, but its response to balloon inflation was quite different. All 7 patients with ventricular arrhythmias showed a significant $(\mathrm{p}<0.01)$

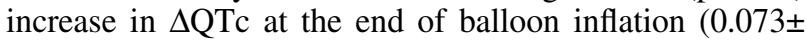
$0.014)$ compared with the pre-inflation control $(0.051 \pm$ $0.015)$, whereas the averaged $\Delta \mathrm{QTc}$ of the patients without arrhythmias decreased significantly at the end of the procedure from $0.063 \pm 0.021$ to $0.039 \pm 0.013(\mathrm{p}<0.01)$. In the non-arrhythmia patient group, the $\Delta$ QTc decreased in 12 patients, increased in 1 and was unchanged in 2 during PTCA (Fig 3). The standard deviation of QTc of the 12 leads increased in the patients with ventricular arrhythmias during the balloon inflation, whereas PTCA decreased the standard deviation of QTc in the patients without arrhythmias, and the reduction tended to return to the pre-inflation level after the termination of the procedure (Fig 4).

The averaged QTa did not change significantly with balloon inflation in any of the leads in either patient group (QTa in leads $\mathrm{V}_{1-3}$ showed an insignificant decrease; Table 2) $\Delta$ QTac during balloon inflation was less in the patients with ventricular arrhythmias than before the balloon inflation, whereas in the patients without arrhythmias $\Delta$ QTac was not affected by PTCA. This change was highly significant $(\mathrm{p}<0.01)$.

With balloon inflation, the ST-segment began to elevate at around $20 \mathrm{~s}$ and peaked within $45 \mathrm{~s}$ after the beginning of myocardial ischemia. Of the 22 patients, 12 had STsegment elevation greater than $0.2 \mathrm{mV}$, and the remaining 10 showed more than $0.3 \mathrm{mV}$ in any of the anterior chest leads during balloon inflation. The averaged maximum STsegment elevation with ventricular arrhythmias was $0.26 \pm$ $0.02 \mathrm{mV}$, and was not significantly different from the STsegment elevation $(0.23 \pm 0.01 \mathrm{mV})$ in patients without arrhythmias $(\mathrm{p}=0.23)$.
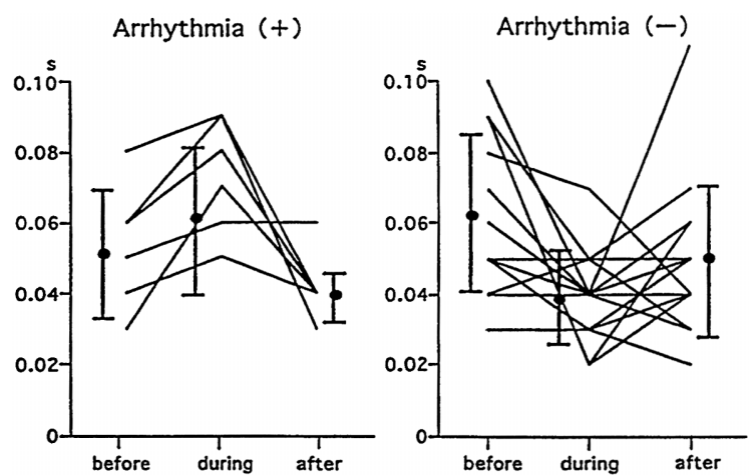

Fig 3. $\Delta \mathrm{QTc}$ before, during and after intracoronary balloon inflation in patients with (Left panel) and without (Right panel) ventricular arrhythmias during the inflation. The balloon inflation significantly $(\mathrm{p}<0.01)$ increased $\Delta \mathrm{QTc}$ in patients with ventricular arrhythmias, whereas in the patients without arrhythmias the procedure decreased the index of repolarization dispersion $(\mathrm{p}<0.11)$. The vertical bars in each measure point shows mean $\pm \mathrm{SD}$.

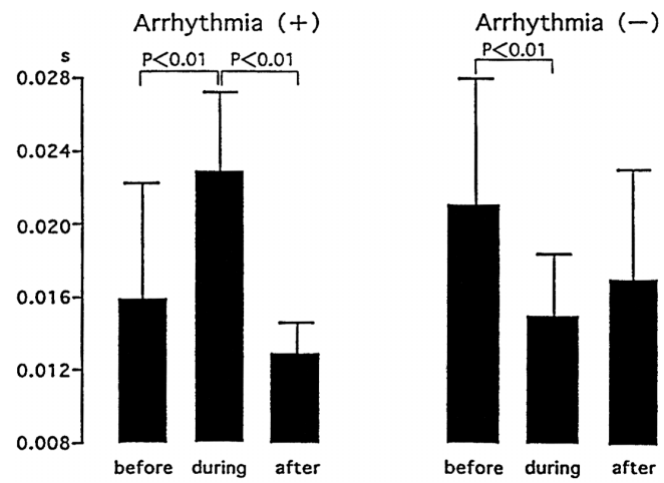

Fig 4. Averaged standard deviation of QTc in the 12-leads before, during and after intracoronary balloon inflation. The standard deviation of QTc increased significantly at the end of the balloon inflation in the patients with ventricular arrhythmias and decreased in the patients without arrhythmias.

\section{Discussion}

This study demonstrated that intracoronary balloon inflation did not modify the averaged QT intervals in patients with stable effort angina, but that an increase in the dispersion of the QT interval during PTCA was associated with ventricular tachyarrhythmias.

PTCA is one of the important methods of treating angina pectoris. Usually the balloon inflation is repeated 3-8 times for each coronary lesion until the lumen of the target segment increases to greater than $50 \%$ of the luminal diameter of the adjacent normal coronary segment. With each balloon inflation, the ST-segment elevated and the QT interval decreased and it was considered that changes in the ST segment and the QT interval were influenced by each previous balloon inflation. To avoid this effect, study data were acquired only during the first balloon inflation.

The changes in the left ventricular diastolic dimension, autonomic nerve tone and endocrine or paracrine secretion caused by transient ischemia may affect the repolarization process of the ventricle. The lack of significant changes in heart rate and blood pressure during PTCA, however, suggest that changes in autonomic nerve tone and endocrine or paracrine secretion were not significantly modified by 
the procedure, although we did not assess these functions in the regional left ventricular wall. As indicated by the similar magnitude of elevation of the ST-segment, the extent of myocardial ischemia during balloon inflation did not differ between the patients with and those without ventricular arrhythmias.

Although ventricular arrhythmias have been frequently observed during and after PTCA, the mechanism(s) of PTCA-induced arrhythmias is still obscure; depolarization disturbance and/or repolarization abnormalities that produce either reentrant ventricular arrhythmias or increased automaticity have been proposed. Postrepolarization refractoriness may also play a role in random reentry during myocardial ischemia; however there are no methods available to measure this clinically. Zareba et al reported the importance of delayed depolarization as a factor for arrhythmic cardiac death as well as heterogeneous ventricular recovery time6 Our finding that the shape and duration of the QRS complex did not change throughout balloon inflation indicates that disturbance of ventricular depolarization may not be responsible for PTCA-induced ventricular arrhythmias.

We used $\Delta$ QTc and standard deviation of QTc (QTcSD) to investigate the dispersion of ventricular repolarization. The results revealed that increases in both indices of repolarization dispersion during intracoronary balloon inflation are associated with ventricular arrhythmias. Dispersion of repolarization has been proposed as a measure of repolarization heterogeneity $1,2,7$ and increased dispersion has been found to be associated with an increased incidence of malignant ventricular arrhythmias ${ }^{8}$ in patients with long QT syndrome, ${ }^{2,9}$ hypertrophic cardiomyopathy 10,11 and congestive heart failure? In ischemic heart disease, many investigators have reported a close relationship between repolarization heterogeneity and malignant ventricular arrhythmias, ${ }^{12,13}$ although Endoh et al failed to find QT dispersion useful as a marker for ventricular arrhythmias in patients with acute myocardial infarction ${ }^{14}$ In patients with a history of sustained ventricular tachycardia (VT) after myocardial infarction, an increased dispersion of the QTc interval was found when compared with the QTc in patients without VT ${ }^{15}$ However, Dristas et al analyzed the ECGs of patients with high-grade ventricular extrasystole without sustained VT after a myocardial infarction and failed to find the same relation!6 Although none of the present 22 patients had a history of spontaneous VT, PTCA-induced ventricular arrhythmias could be related to ventricular repolarization heterogeneity. This study shows that QT dispersion is a valid index of PTCA-induced ventricular arrhythmias. We consider that it may also be a valid measure in spontaneous ventricular arrhythmias, although this would be difficult to study. A stepwise reduction of QT dispersion with an increasing number of balloon inflations and deflations was reported in patients with and without previous myocardial infarction ${ }^{17}$ which suggests that ischemic preconditioning increases the electrophysiological stability of the myocardium, although none of those patients showed arrhythmic events during the course of the procedure and we cannot understand why none of the 47 patients of their study had ventricular arrhythmias during PTCA; it is not unusual that a short-lasting myocardial ischemia causes ventricular arrhythmias. Because we analyzed the QT interval before and after the first inflation to avoid the effect of ischemic preconditioning on ventricular repolarization, it is hard to interpret the effect of ischemic preconditioning.
Perkiomaki et al divided the QT interval of patients with ventricular arrhythmias after a previous myocardial infarction into 2 components: the interval from the onset of the QRS complex to the apex of the T wave (QTa) and the interval from the apex to the end of the T wave (Te) $!^{13}$ They found that broad dispersion of the total QT interval was mainly caused by dispersion of the Te rather than QTa interval. The present results are comparable to the Perkiomaki's report; the dispersion of QTac was rather less during the balloon inflation than before PTCA. Thus, the regional difference of the latter part of ventricular repolarization is mainly responsible for the inhomogeneities in myocardial recovery time, and may be important for arrhythmogenesis.

\section{Acknowledgment}

We are grateful to Dr JL Bossian for valuable discussion regarding this report.

\section{References}

1. Cowan JC, Yusoff K, Moore M, Amos PA, Gold AE, Bourke JP, et al: Importance of lead selection in QT interval measurement. Am J Cardiol 1988; 61: 83-87

2. Day CP, McComb JM, Campbell RWF: QT dispersion; An indication of arrhythmia risk in patients with long QT intervals. Br Heart J 1990; 63: $342-344$

3. Barr CS, Naas A, Freeman M, Lang CC, Struthers AD: QT dispersion and sudden unexpected death in chronic heart failure. Lancet 1994; 343: 327-329

4. Buja G, Miorelli M, Turrini P, Malacini P, Nava A: Comparison of QT dispersion in hypertrophic cardiomyopathy between patients with and without ventricular arrhythmias and sudden death. Am J Cardiol 1993; 72: $973-976$

5. Higham PD, Hilton CJ, Aitcheson JD, Furniss SS, Bourke JP, Campbell RWF: QT dispersion: A measure of underlying dispersion of ventricular recovery? (abstract) Eur Heart J 1993; 14: 86

6. Zareba W, Moss AJ, le Cessie S: Dispersion of ventricular repolarization and arrhythmic cardiac death in coronary artery disease. Am J Cardiol 1994; 74: 550-553

7. Merri M, Benhorin J, Alberti M, Locati E, Moss AJ: Electrocardiographic quantitation of ventricular repolarization. Circulation 1989; 80: $1301-1308$

8. Pye M, Quinn AC, Cobbe SM: QT interval dispersion: A non-invasive marker of susceptibility of arrhythmia in patients with sustained ventricular arrhythmias? Br Heart J 1994; 71: $511-514$

9. Linker NJ, Colonna P, Kekwick CA, Till J, Camm AJ, Ward DE: Assessment of QT dispersion in symptomatic patients with congenital long QT syndromes. Am J Cardiol 1992; 69: 634-638

10. Miorelli M, Buja G, Melacini P, Fasoli G, Nava A: QT-interval variability in hypertrophic cardiomyopathy patients with cardiac arrest. Int J Cardiol 1994; 45: $121-127$

11. Dritsas A, Gillian D, Nihoyannopoulos P, Oakley CM: Amiodarone reduces QT-dispersion in patients with hypertrophic cardiomyopathy. Int J Cardiol 1992; 36: 345-349

12. Dristas A, Sbarouni E, Oakley CM, Cleland JGF: Is QT interlead variability an arrhythmogenic marker? Br Heart J 1992; 68: 116

13. Perkiomaki JS, Koistinen MJ, Yli-Mayry S, Huikuri HV: Dispersion of QT interval in patients with and without susceptibility to ventricular tachyarrhythmias after previous myocardial infarction. J Am Coll Cardiol 1995; 26: 174-179

14. Endoh Y, Kasanuki H, Ohnishi S, Uno M: Unsuitability of QT dispersion as a marker for ventricular arrhythmias and cardiac sudden death after acute myocardial infarction. Jpn Circ J 1999; 63: 467-470

15. Bashir Y, Farre T, Camm AJ: Increased QT dispersion in survivors of acute myocardial infarction who subsequently develop ventricular arrhythmias or sudden death. Pacing Clin Electrophysiol 1990; 13: 561

16. Dristas A, Sbarouni E, Nihoyannopoulos P, Cleland J, Oakley C: Relation of QTc interval and QTc dispersion to ventricular arrhythmia in patients with previous myocardial infarction. Eur Heart J 1992; 13: 344

17. Okishige K, Yamashita K, Yoshinaga H, Azegami K, Sato T, Goseki $\mathrm{Y}$, et al: Electrophysiologic effects of ischemic preconditioning on QT dispersion during coronary angioplasty. J Am Coll Cardiol 1996; 28: $70-73$ 